\title{
Some Approaches in Online Misinformation Analysis
}

\author{
Uyiosa Omoregie \\ August 2021 \\ Avram Turing, Guelph, ON, Canada \\ uyiosa.omoregie@avramturing.com
}

The complex nature of the 'infodemic' problem requires a combination of different approaches to analysing online information disorder. Approaches which emphasize analytical and critical thinking are important but have shortcomings. When analysing complex phenomena like conspiracy theories a 'systems' approach is more effective to reveal root causes of information disorder, provide actionable insight and long-term solutions. Zhang et al. [1] emphasize the multifaceted nature of the misinformation challenge and varied initiatives and solutions proffered against the menace.

An 'infodemiological' approach to misinformation analysis makes use of network analysis. This approach seeks to understand the spread of misinformation the way an epidemiologist studies disease [2]. Some analysts use a cybersecurity approach: viewing online misinformation as a national security challenge. Equipped with cybersecurity tools, they track misinformation the way they would track malware, to prevent "the hacking of people's beliefs" [3][4]. Popat et al. [5] employ a linguistic approach to misinformation analysis by assessing the credibility of emerging claims online. They demonstrate the importance of the language style of textual claims online. Increasingly important is the social justice/power structures approach to online misinformation analysis [6] [7] [8]. This approach emphasizes the role power differentials within society play in certain kinds of disinformation that amplify racial stereotypes and increase xenophobia, misogyny and anti-Semitism.

A Wittgensteinian approach to misinformation analysis [9] [10] presents a model inspired by the framework of the early philosophy of Ludwig Wittgenstein. This approach is based on 'analysis' or analytical thinking, which could be differentiated from 'criticism' or critical thinking. Analysis involves decomposition of information to simpler elements for clarity and better understanding, the opposite of 'synthesis' [11]. Critical thinking is more focused on the evaluation of information, interpreting it and making an informed judgment [12]. Analytical and critical approaches complement each other. Misinformation online manifests in different forms, a combination of different approaches to tackle it is required for effective solutions. Cook et al. [13] present a critical thinking approach to deconstruct misinformation. They identify reasoning fallacies, based on argument structure, to refute common misinformation about climate change espoused by denialists. This approach is comprehensive and was successful when applied to 42 claims commonly peddled by denialists.

Fact-checking as a misinformation prevention strategy has limitations [14]. Certain types of misinformation disorder are complex and providing 'facts' alone may not change beliefs in adherents, particularly when deeply-held beliefs are involved. Sometimes the opposite effect of strengthening the false belief occurs. This has led to the strategy of trying to prevent or neutralize misinformation through 'inoculation' or 'prebunking' [15]. Prebunking or inoculation involves exposing the flawed argumentation techniques of misinformation to prepare online content consumers against future misinformation. The importance of timing when correcting 'fake news' has been emphasized in a study to demonstrate the effectiveness of 'debunking'[15]. 'Debunking' was defined as fact-checks after misinformation exposure, 'labeling' (fact-checks of information presented during exposure) and 'prebunking' (fact-checks before exposure). 
Breaking down arguments into 'logical atoms' [9] a form of reductionism will not be effective for complex phenomena like conspiracy theories. Analysing conspiracy theories is best done using a systems approach or systems thinking [16]. Systems thinking is a way of viewing the world in all its complexity: a holistic (as opposed to reductionist) worldview that emphasises learning and the search for high leverage points to facilitate lasting positive change [17] [18]. The systems approach is not a linear approach or "open-loop, event-oriented view of the world" (see Figure 1 below) but rather a "feedback view of the world" (see Figure 2 below) [17].

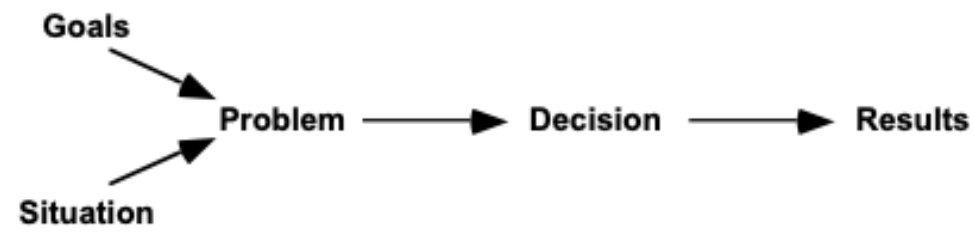

Figure 1: The open-loop, event-oriented view of the world [17]

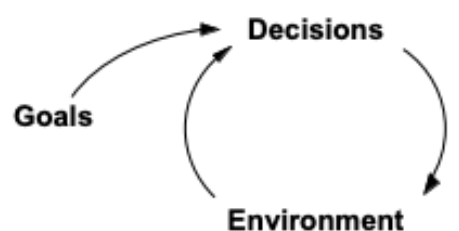

Our decisions alter our environment, leading to new decisions,

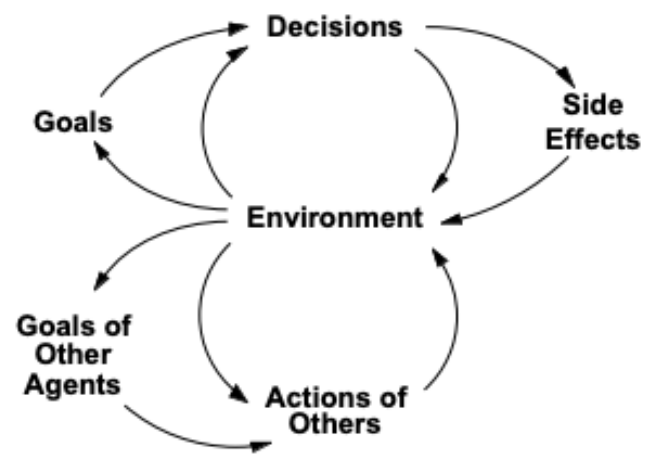

but also triggering side effects, delayed reactions, changes in goals and interventions by others. These feedbacks may lead to unanticipated results and ineffective policies.

Figure 2: The feedback view of the world [17]

The Walters Foundation [19] describes the following "Habits of Systems Thinkers": 1. Seek to understand the big picture; 2 . Observe how elements within the system change over time, generating patterns and trends; 3. Recognize that a system's structure (elements and 
interactions) generates behaviour; 4. Identify the circular nature of complex cause-andeffect relationships; 5 . Surface and test assumptions; 6 . Change perspective to increase understanding; 7. Consider an issue fully and resist the urge to come to a quick conclusion; 8. Consider how mental models affect current reality and the future; 9. Use understanding of system structure to identify possible leverage actions; 10. Find where unintended consequences emerge; 11 . Recognize the impact of time delays when exploring cause-andeffect relationships; 12. Check results and change actions if needed: "successive approximation."

Ammara et al. [16] use a systems approach to analyse misinformation: an emphasis on identifying roots causes, provide actionable insight and anticipating long-term consequences. They reveal that incorrect labelling of news by fact-checkers could lead to more scepticism of news credibility as a whole. They also discover that a focus on improvements in artificial intelligence (Al) for detection of fake news could lead to similar technology being used to generate more fake news.

\section{References}

[1] Zhang, A, Ranganathan, A, Metz, S, et al. A structured response to misinformation: defining and annotating credibility indicators in news articles. The 2018 Web Conference Companion. https://dl.acm.org/doi/10.1145/3184558.3188731

[2] Freedman, D. As QAnon conspiracy theories draw new believers, scientists take aim at misinformation pandemic. Newsweek, https://www.newsweek.com/2020/10/23/qanonconspiracy-theories-draw-new-believers-scientists-take-aim-misinformation-pandemic1538901.html

[3] Kehrt, S. One data scientist's quest to quash misinformation. Wired, https://www.wired.com/story/data-scientist-cybesecurity-tools-quash-misinformation/ (2020, accessed 27 September 2020).

[4] Schapals, A. Fake news. J. Pract. 2018;12, 976-985.

[5] Popat, K, Mukherjee, S, Strötgen, J, Weikum, G. Where the truth lies: explaining the credibility of emerging claims on the web and social media. In Proceedings of the 26th International Conference on World Wide Web Companion 2017 Apr 3 (pp. 1003-1012). https://dl.acm.org/doi/10.1145/3041021.3055133,

[6] Noble, S. Algorithms of Oppression: How Search Engines Reinforce Racism. New York: NYU Press. 2018.

[7] Marwick, A, Kuo, R, Cameron, S and Weigel, M. Critical disinformation studies: a syllabus. Center for Information, Technology, \& Public Life (CITAP), University of North Carolina at Chapel Hill, http://citap.unc.edu/critical-disinfo (2021, accessed 28 April 2021).

[8] Kreiss, D. "Social Media and Democracy: The State of the Field, Prospects for Reform," edited by Nathaniel Persily and Joshua A. Tucker. Int. J. Press/Politics. 2021; 26(2):505-512.

[9] Omoregie, U. Online misinformation analysis and information quality theory. SocArXiv, https://osf.io/preprints/socarxiv/82swk/ 
[10] Omoregie, U. Misinformed about the information age: The existential crisis of online social media. Business Day, https://businessday.ng/opinion/article/misinformed-aboutthe-information-age-the-existential-crisis-of-online-social-media/

[11] Beaney, M. Analysis. The Stanford Encyclopedia of Philosophy, https://plato.stanford.edu/entries/analysis/

[12] The Peak Performance Center. Analytical thinking and critical thinking, https://thepeakperformancecenter.com/educational-learning/thinking/criticalthinking/analytical-thinking-critical-thinking/

[13] Cook, J, Ellerton, P, Kinkead, D. Deconstructing climate misinformation to identify reasoning errors. Environ. Res. Lett. 2018; 13(2):024018.

[14] Cook, J, Lewandowsky, S and Ecker, UK, et al. Neutralizing misinformation through inoculation: exposing misleading argumentation techniques reduces their influence. Plos One 2017; 12:5 e0175799.

[15] Brashier, N, Pennycook, G, Berinsky, AJ, et al. Timing matters when correcting fake news. P Natl Acad Sci 2021; 118: 5 e2020043118

[16] Ammara, U, Bukari, H and Qadir, J. Analyzing misinformation through the lens of systems thinking. Proceedings of the 2020 Truth and Trust Online (TTO 2020); 55-63.

[17] Sterman, JD. Systems dynamics: system thinking and modelling for a complex world. Massachusetts Institute of Technology Engineering Systems Division, Working Paper Series ESD-WP-2003-01.13-ESD Internal Symposium 2002, https://dspace.mit.edu/bitstream/handle/1721.1/102741/esd-wp-200301.13.pdf? sequence $=1 \&$ is Allowed $=y$

[18] Omoregie, U. Management thinking for complex issues: review of two contrasting perspectives. FMR. 2017; 1:96-105.

http://www.isaacpub.org/images/PaperPDF/FMR_100020_2017091216050950335.pdf

[19] Waters Foundation. Habits of a systems thinker, https://ttsfilestore.blob.core.windows.net/ttsfiles/habits-single-page-2020.pdf 